\title{
Hyperactivity in the Gunn rat model of neonatal jaundice: age-related attenuation and emergence of gait deficits
}

\author{
John A. Stanford', Jeffrey M. Shuler', Stephen C. Fowler' ${ }^{2}$ Kimberly G. Stanford', Delin Ma', Douglas C. Bittel' \\ Jean-Baptiste Le Pichon ${ }^{4-6}$ and Steven M. Shapiro ${ }^{4-6}$
}

\begin{abstract}
BACKGROUND: Neonatal jaundice resulting from elevated unconjugated bilirubin occurs in $60-80 \%$ of newborn infants. Although mild jaundice is generally considered harmless, little is known about its long-term consequences. Recent studies have linked mild bilirubin-induced neurological dysfunction (BIND) with a range of neurological syndromes, including attention-deficit hyperactivity disorder. The goal of this study was to measure BIND across the lifespan in the Gunn rat model of BIND.
\end{abstract}

METHODS: Using a sensitive force plate actometer, we measured locomotor activity and gait in jaundiced (jj) Gunn rats versus their nonjaundiced ( $\mathrm{Nj}$ ) littermates. Data were analyzed for young adult (3-4 mo), early middle-aged (9-10 mo), and late middle-aged (17-20 mo) male rats.

RESULTS: $j$ j rats exhibited lower body weights at all ages and a hyperactivity that resolved at 17-20 mo of age. Increased propulsive force and gait velocity accompanied hyperactivity during locomotor bouts at 9-10 mo in jj rats. Stride length did not differ between the two groups at this age. Hyperactivity normalized, and gait deficits, including decreased stride length, propulsive force, and gait velocity, emerged in the 17-20-moold jj rats.

CONCLUSION: These results demonstrate that, in aging, hyperactivity decreases with the onset of gait deficits in the Gunn rat model of BIND.

$\mathbf{N}$ eonatal jaundice resulting from elevated levels of unconjugated bilirubin occurs in up to $85 \%$ of newborns (1). When unconjugated bilirubin levels exceed the binding capacity of albumin, free bilirubin can enter the brain where it targets discrete nuclei, including the globus pallidus, cochlear nucleus and inferior colliculus, hippocampus, and cerebellum. Free bilirubin is toxic at elevated levels, resulting in a constellation of devastating and permanent neurological effects known as kernicterus (2). Little is known, however, about the long-term consequences of more subtle levels of neonatal jaundice. Emerging evidence supports a subtle kernicteric syndrome that is referred to as bilirubin-induced neurological dysfunction (BIND). BIND has been linked to a range of neuropsychiatric syndromes that persist into adulthood, including attention-deficit hyperactivity disorder (ADHD), autism, and schizophrenia (3-6). Even with modest comorbidities, the incidence of neonatal jaundice justifies further exploration of links between these disorders and BIND.

The goal of this study was to measure locomotor activity and gait across the lifespan in the Gunn rat model of BIND (7). Gunn rats are genetically deficient of uridine $5^{\prime}$-diphosphoglucuronosyl transferase, the liver enzyme responsible for bilirubin conjugation and clearance. These rats become jaundiced (jj) soon after birth and exhibit physical, neurological, and behavioral abnormalities. Their blood bilirubin levels peak at $16 \mathrm{~d}$ postnatal age and fall to lower but elevated levels throughout the rest of their lives (8-11). Although Gunn rats model classical kernicterus when administered sulfa during discrete postnatal time periods $(9,12-14)$, untreated Gunn rats are a model of BIND. The most apparent abnormalities during the early postnatal days are their smaller size and hyperactive phenotype (15). More subtle and variable deficits include attention and learning deficits, deficits in social behavior, and anecdotal reports of ataxia $(6,15,16)$. Anatomical and histopathological studies report cerebellar hypoplasia, hippocampal mitochondrial abnormalities, and alterations in catecholamines in the basal ganglia and cerebellum of Gunn rats (16-18). Disruptions in the circuitry through which these brain regions interact likely underlie the behavioral deficits that have been reported in this model (reviewed in ref. (5)). Although jaundice peaks during early postnatal days in Gunn rats as it does in humans, we are studying these rats through adulthood and aging to determine long-term sequelae of BIND.

\section{RESULTS}

Overall, jj rats maintained significantly lower body weights than their nonjaundiced $(\mathrm{Nj})$ counterparts at all ages (Figure 1a). Locomotor activity was significantly greater in the $\mathrm{jj}$ rats at 3-4 mo during the initial 15-min testing sessions, $F(1,6)=$ 39.656, $P=0.001$ (Figure 1b). This difference between groups persisted across $6 \mathrm{~d}$ of testing. When the older groups were

'Department of Molecular and Integrative Physiology, University of Kansas Medical Center, Kansas City, Kansas; ${ }^{2}$ Department of Pharmacology and Toxicology, University of Kansas, Lawrence, Kansas; ${ }^{3}$ The Ward Family Heart Center, Children's Mercy Hospital, Kansas City, Missouri; ${ }^{4}$ Department of Neurology, Children's Mercy Hospital, Kansas City, Missouri; ${ }^{5}$ Departments of Neurology and Pediatrics, University of Kansas Medical Center, Kansas City, Kansas; ${ }^{6}$ Department of Pediatrics, University of Missouri-Kansas City, Kansas City, Missouri. Correspondence: John A. Stanford (jstanford@kumc.edu) 
a

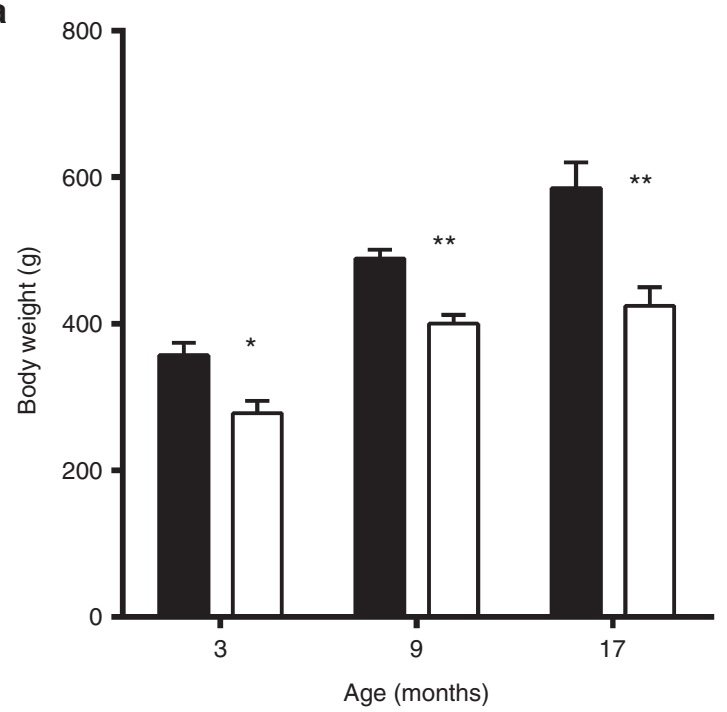

b

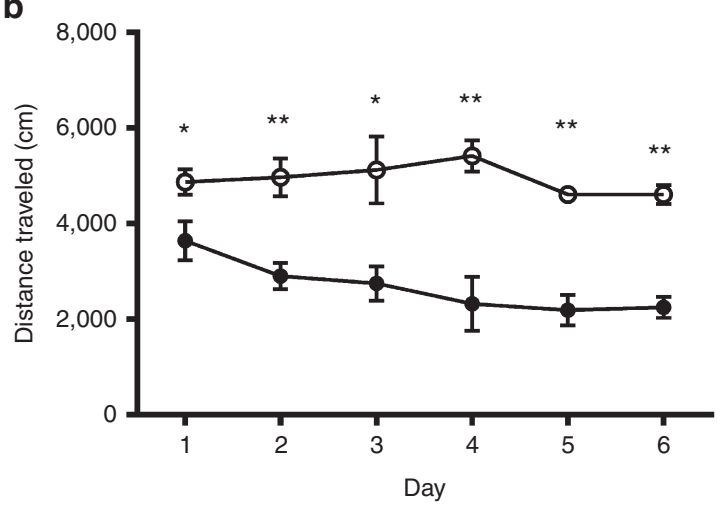

Figure 1. Body weight and locomotor activity as a function of group and age. (a) At each age, Nj rats' (filled bars) body weights exceeded those of jj rats (open bars). (b) Distance traveled during 15-min, weekly testing sessions. Rats in the jj group (open symbols) exhibited greater locomotion than $\mathrm{Nj}$ rats (filled symbols) on each testing day. ${ }^{*} P<0.05$; ${ }^{* *} P<0.01$.

tested, locomotor activity remained higher in the 9-10-mo-old jj rats, $F(1,6)=8.117, P<0.05$, but normalized by $17-20 \mathrm{mo}$, $F(1,13)=0.137, P=0.72$ (Figure 2a).

Neither the number of runs, the duration of runs, the length of individual runs, or stride rate differed significantly as a function of group or age (Table 1). However, for stride length, run velocity, and within-run force range, differences emerged between the groups. Stride length was similar between the 9-10-mo-old jj and $\mathrm{Nj}$ rats, $F(1,6)=1.074, P=0.34$, but was significantly decreased in the 17-20-mo-old jj rats, $F(1,14)$ $=13.22, P<0.01$ (Figure $2 \mathbf{b}$ ). Run velocity was significantly greater in the 9-10-mo-old jj rats, $F(1,6)=6.061, P<0.05$, but was decreased in the older jj group, $F(1,15)=10.411, P<0.01$ (Figure 2c). Within-run force range was similarly affected, as propulsive force was greater in 9-10-mo-old rats, $F(1,6)$ $=27.320, P<0.01$, but diminished in 17-20-mo-old jj rats, $F(1,11)=9.104, P<0.05$ (Figure 2d).

\section{DISCUSSION}

Overall, jj rats exhibited decreased body weights that persisted across their lifespan, and a hyperactive phenotype that resolved in late middle age. Gait abnormalities, such as decreased stride length, decreased stride velocity, and decreased propulsive force, emerged in the 17-20-mo-old jj group. These results are consistent with reported effects of neonatal jaundice on hyperactivity and on basal ganglia, hippocampal, and cerebellar pathology in the Gunn rat model.

The hyperactive phenotype exhibited by the jj rats was consistent with previous findings in $60-\mathrm{d}$-old jj rats (15). Our results extend these findings to older adulthood. We also report for the first time that the hyperactivity produced by neonatal jaundice resolves at late middle age (17-20 mo) in the Gunn rat model. Although the neural mechanisms underlying this phenomenon have not been identified, interactions between the basal ganglia and cerebellum may play a role (5). Disruptions of the cortico-cerebellar-basal ganglia circuit would adversely affect appropriate behavioral response selection and inhibition. It is tempting to attribute the decreased hyperactivity in the aged jj rats to normal age-related decreases in basal ganglia function (19-21). However, this hypothesis is not supported by the fact that locomotor activity in $\mathrm{Nj}$ rats did not decrease between the 9-10-mo-old and the 17-20-mo-old groups. Because agerelated decreases in locomotor activity are typically present by 18 mo of age in rats (19), our findings suggest potential behavioral abnormalities in $\mathrm{Nj}$ littermates. The addition of a control group with full uridine $5^{\prime}$-diphospho-glucuronosyl transferase activity is necessary to test this hypothesis. Alternatively, there is pathological evidence for damage to the hippocampus in Gunn rats (18). Lesions of the ventral hippocampus result in hyperactivity in adult rats (22-25), so it is possible that toxic effects of unconjugated bilirubin in the hippocampus contributed to-if not accounted for-the hyperactive phenotype. It is well documented that hyperactivity following damage to the hippocampus during early postnatal periods is delayed, emerging in young adult rats $(23,24)$. Although speculative, these previous findings are consistent with hippocampal involvement in the hyperactivity we measured in $\mathrm{jj}$ rats. Further studies examining neural activity in affected structures are clearly warranted to test these hypotheses.

Gait deficits, including decreased stride length, are present in a number of neurological conditions with different underlying pathologies, including Parkinson's disease (26), Huntington's disease (27), and amyotrophic lateral sclerosis (28). Although lesions in the areas affected in each of these conditions likely disrupt motor-related circuits through the cerebellum, basal ganglia, and cortex, anatomical and pathological studies in Gunn rats support a primary role for cerebellar dysfunction in the gait deficits we observed (16). The fact that we did not detect significant differences in stride length or other gait parameters in our 9-10-mo-old group was surprising but consistent with studies that have quantified gait in this model (29). Although other groups have reported a wobbly gait or ataxia in young Gunn rats $(15,30,31)$, these reports have been observational. We also observed and quantified a wobbly gait in some, but not all, of the younger Gunn rats in our study (data not shown). Gait abnormalities and motor incoordination might be expected earlier in humans, but the 
a

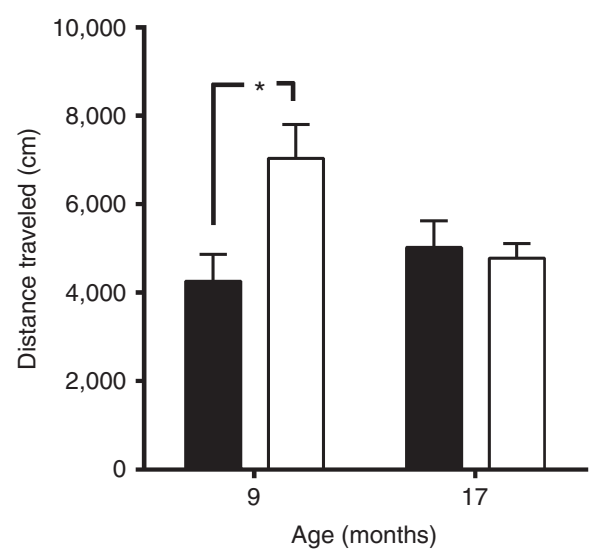

C

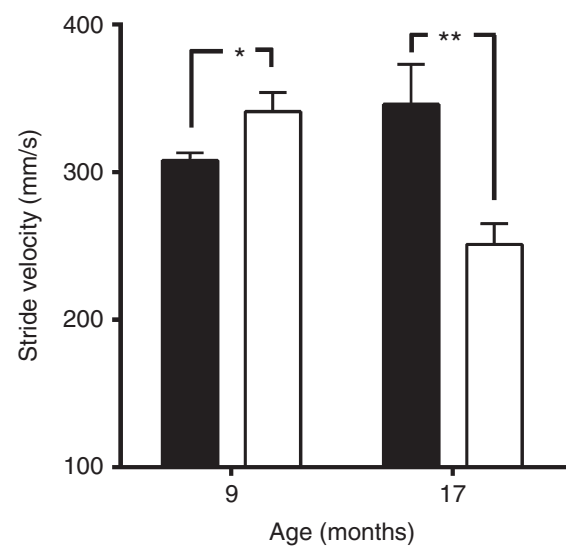

b

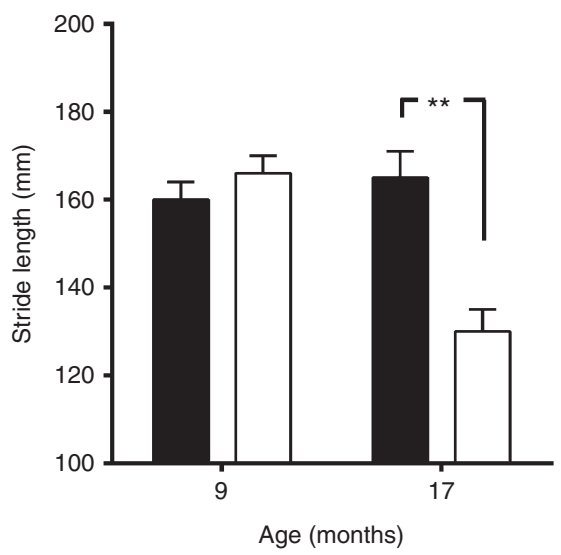

d

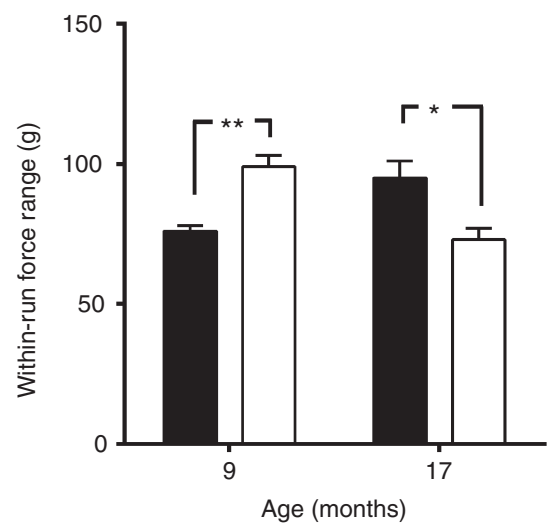

Figure 2. Distance traveled and gait measures in adult vs. late middle-aged $\mathrm{Nj}$ (filled bars) vs. jj (open bars) rats. (a) Distance traveled was greater for the 9-10 mo-old jj rats, but this effect was not present at 17-20 mo. (b) Stride length was diminished in the 17-20-mo-old jj group. Compared to Nj rats, (c) run velocity and (d) within-run force range were both greater in the 9-10-mo-old jj group but diminished in the 17-20-mo-old jj group. ${ }^{*} P<0.05$; $* * P<0.01$.

Table 1. Gait measures that did not differ significantly between groups

\begin{tabular}{lcccc}
\hline Measure & $9-10 \mathrm{mo} \mathrm{Nj}$ & $9-10 \mathrm{mojj}$ & $17-20 \mathrm{mo} \mathrm{Nj}$ & $17-20 \mathrm{mojj}$ \\
\hline Runs $(n)$ & $10 \pm 2$ & $13 \pm 2$ & $14 \pm 2$ & $10 \pm 2$ \\
Duration of runs $(\mathrm{s})$ & $1.28 \pm 0.05$ & $1.16 \pm 0.03$ & $1.22 \pm 0.13$ & $1.4 \pm 0.05$ \\
Run length $(\mathrm{mm})$ & $385 \pm 8$ & $386 \pm 14$ & $407 \pm 17$ & $344 \pm 16$ \\
Stride rate $(\mathrm{Hz})$ & $1.91 \pm 0.02$ & $2.06 \pm 0.11$ & $2.10 \pm 0.17$ & $1.99 \pm 0.04$ \\
\hline
\end{tabular}

Data are represented as means \pm SEM.

timing is different in Gunn rats than most humans with hyperbilirubinemia. In Gunn rats, bilirubin peaks at about $12 \mathrm{mg} /$ $\mathrm{dl}$ at $16 \mathrm{~d}$ of age (9) but remains elevated throughout life. The abnormalities we found may be a delayed effect of the neonatal peak, but perhaps the chronic levels are in part responsible for later responses. In addition, concurrent illness (e.g., viral infections) can exacerbate bilirubin neurotoxicity in jj Gunn rats with minimal signs of neurotoxicity. Over time, these may have contributed to the later emerging symptoms. We did not measure cerebellar damage or hypoplasia, but these phenomena are features in these rats as early as 7-10 d of postnatal age $(32,33)$. It is possible that compensation accounted for the lack of gait abnormalities in our 9-10-mo-old group. For example, previous studies demonstrate that aging has a detrimental effect on cerebellar specific motor learning and exacerbates ataxia resulting from cerebellar lesions (34-36). For example, anatomical reorganization and functional recovery is greater following cerebellar damage in the neonate than in the adult rat (34,35). Although 9-10-mo-old rats are adults, the cerebellar lesions present in Gunn rats are likely less severe than those produced experimentally in the cited studies. Despite the presence of visible ataxia in some of our jj rats throughout their lifespan, our results suggest that the toxic effects of neonatal jaundice on cerebellar circuits may go undetected until these circuits are further perturbed by the effects of normal aging (e.g., decreased $\beta$-adrenergic receptor function, impaired motor learning) on cerebellar function (36).

Previous studies have cited comorbidities between neonatal jaundice and various neuropsychiatric disorders, including ADHD (3-6). Damage to the globus pallidus, hippocampus, and cerebellum in BIND is consistent with clinical findings of abnormalities in each of these regions in $\operatorname{ADHD}(37,38)$. Our findings support a link between BIND and ADHD in that, as in the clinical situation, hyperactivity generally resolves with increased age $(3,39)$. We believe that the human correlate for what we measured is hyperactivity or perhaps ADHD. 

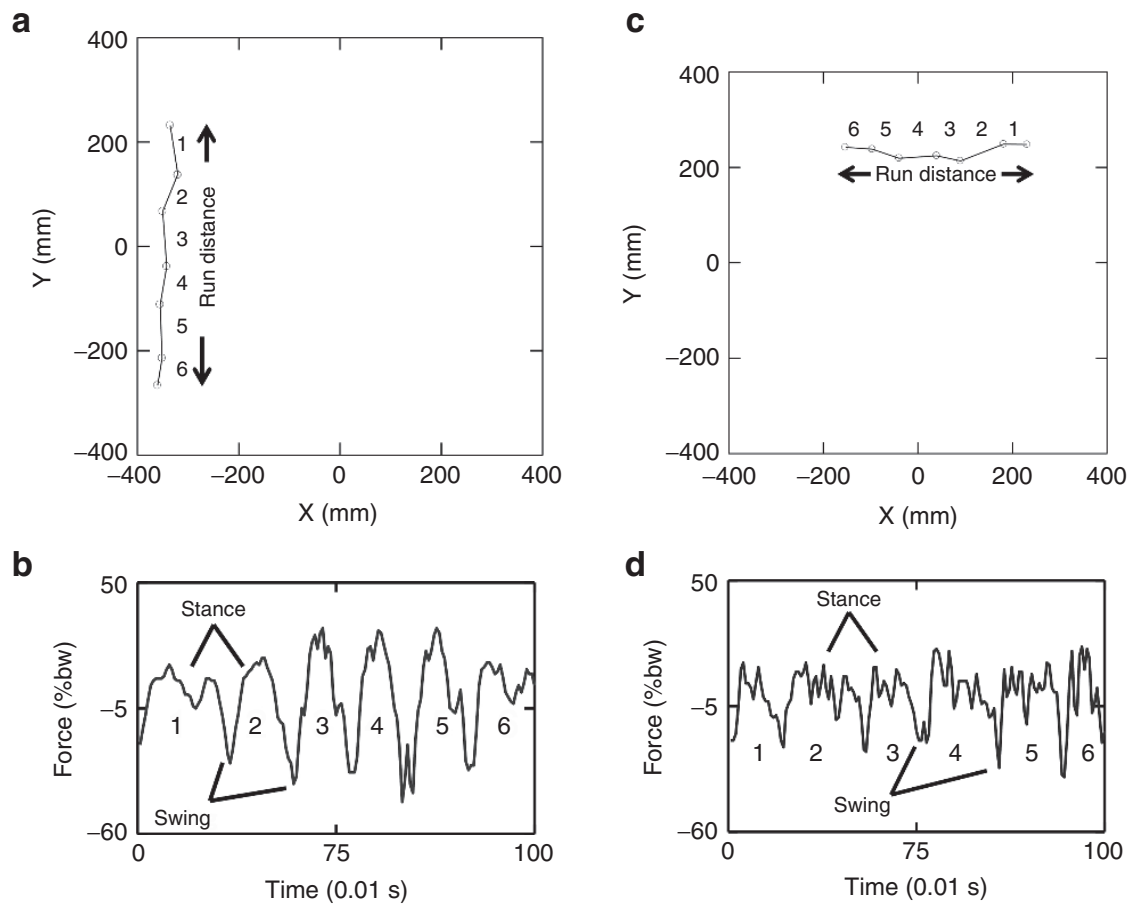

Figure 3. Changes in center of force and raw force-time records illustrating one run from one representative 20-mo-old Nj rat (left) and one representative 20-mo-old jj rat (right). (a) Center of force measures indicate the run distance for the $\mathrm{Nj}$ rat. Numbers $1-6$ along the relatively straight line along the $y$ axis refer to the sequential order of half strides. (b) The graph shows changes in the Nj rat's vertical force during the run shown in a. The numbers $1-6$ refer to the six half strides depicted in a. Two examples of stance and swing phases are indicated. (c) Center of force measures indicate the run distance for the jj rat. Numbers 1-6 along the relatively straight line along the $x$ axis refer to the sequential order of half strides. (d) The graph shows changes in the jj rat's vertical force during the run shown in panel c. The numbers 1-6 refer to the six half strides depicted in c. Two examples of stance and swing phases are indicated.

While speculative, we hypothesize that BIND results in an ADHD phenotype in young Gunn rats until it is attenuated by gait abnormalities due to cerebellar dysfunction in aging. One potential weakness of our study was the lack of bilirubin measurements in our $\mathrm{jj}$ and $\mathrm{Nj}$ groups. The value of total (vs. unbound, or free) bilirubin in predicting BIND is questionable, however (40), and measurement techniques for free bilirubin are in their infancy. In addition, we did not measure auditory dysfunction in our study. Changes in brainstem auditory evoked potentials have been measured in neonatal and young adult Gunn rats (14) and were likely present in our rats. To our knowledge, auditory function has not been measured in older Gunn rats. Given the significant incidence of neonatal jaundice, additional studies are needed to further validate this model and determine how BIND relates to ADHD and ataxia in the elderly.

\section{METHODS}

Animals

Male $\mathrm{jj}$ or $\mathrm{Nj}$ rats were studied at 3-4 mo of age, 9-10 mo of age, or 17-20 mo of age. We limited our study to male rats due to the increased risk of hyperbilirubinemia in males (41). We did not generate an NN strain with full uridine $5^{\prime}$-diphospho-glucuronosyl transferase activity to use as controls as $\mathrm{Nj}$ rats have not been shown to exhibit physical or neurological abnormalities. For the 3-4-mo-old and 9-10-mo-old groups, we used four $\mathrm{jj}$ rats and four $\mathrm{Nj}$ rats. For the 17-20-mo-old groups, we used $12 \mathrm{jj}$ rats and $4 \mathrm{Nj}$ rats (we tested more jj rats because we kept more of them in our colony longer than their $\mathrm{Nj}$ littermates). Distance traveled was measured in all groups, but gait was only measured in the 9-10-mo-old and 17-20-mo-old groups because meticulous paw print analyses have revealed that younger $\mathrm{jj}$ and $\mathrm{Nj}$ rats do not differ with regard to stride length (29). Animals were housed in the University of Kansas Medical Center's AAALACaccredited animal facility, and all procedures adhered to the policies put forth in the Guide for the Care and Use of Laboratory Animals (2011) and were approved by the University of Kansas Medical Center Institutional Animal Care and Use Committee.

\section{Locomotor Activity and Gait}

The force plate actometer was constructed in-house and had an $80 \times 80 \mathrm{~cm}$ floor that recorded ground reaction forces as rats moved individually around the arena for $60 \mathrm{~min}$. The animal's center of force was quantified using Cartesian coordinates derived from the locations of the four force sensors that were rigidly attached to each corner of the actometer surface (see ref. (42)). Distance traveled was quantified based on changes in the rat's center of force during each session. Force transducer voltages were digitized at the rate of 100 samples/s, and ground reaction forces were expressed as percent of body weight. We initially analyzed distance traveled in the 3-4-moold groups across six 15-min sessions 1 wk apart. For the older groups, sessions were $60 \mathrm{~min}$ in duration to allow for measurements of gait patterns made possible by the large size of the actometer. A scrolling graphics program written in-house was used to visualize, count, and record consecutive force-time variations that constituted half strides. Temporally aligned on the same screen with the force data were the rat's location coordinates, which allowed the observer to identify and record only rhythmic, long, straight, fast, continuous runs and their associated force time series (see two examples depicted in Figure 3). Each cycle is a half stride. In the figure, the numbers 1 through 6 give the temporal order of half stride production. A rhythmic sequence of four or more half strides constituted a run where diagonally opposite feet act in unison to produce alternating stance and swing phases (Figure 3). In order to be considered suitable for quantitation, four 
or more half strides (i.e., two full strides was the minimum) had to be discerned by the program user. Additional criteria for including a run in the data set for estimation of gait parameters were: (i) A run had to be reasonably straight. This was judged by the program user by noting the lack of curvature in $\mathrm{X}(t)$ and/or $\mathrm{Y}(t)$ during the run sequence of half strides. This criterion ensured that variation in rhythm and other gait parameters would not be the result of changing direction at corners, etc. (ii) A run had to be expressed as a continuous sequence of half strides without any pausing in locomotion. (iii) Rhythmic sequences of force variation, such as those produced by grooming or scratching were easily excluded from scoring because these in-place behaviors were not accompanied by locomotion. These data, which were saved in an Excel file, were subjected to further processing in SYSTAT (Systat Software, San Jose, CA) in order to calculate the number of runs, duration of run, stride length (run distance/number of strides), stride rate (strides/s), velocity (run distance/run duration), within-run force range (maximum - minimum force), run distance (measured directly using scrolling graphics program), and number of half strides/run (measured directly using scrolling graphics program). Each of these parameters was then averaged across runs for each rat. ANOVA was used to compare $\mathrm{jj}$ vs. $\mathrm{Nj}$ groups. ANOVAs were conducted separately for each age group, and $P \leq 0.05$ was considered statistically significant.

\section{ACKNOWLEDGMENT}

The authors thank Stan Fernald for assistance with images.

\section{STATEMENT OF FINANCIAL SUPPORT}

This work was supported by Children's Mercy Hospital, Kansas City, MO, USA; American Heart Association grant 11GRNT7380047; National Institutes of Health grant AG035982; and National Institutes of Health grant HD02528.

Disclosure: The authors have no financial ties to products in the study or potential/perceived conflicts of interest.

\section{REFERENCES}

1. Watchko JF, Tiribelli C. Bilirubin-induced neurologic damage-mechanisms and management approaches. N Engl J Med 2013;369:2021-30.

2. Shapiro SM. Kernicterus. In: Stevenson DK, Maisels MJ, Watchko JF, eds. Care of the Jaundiced Neonate. New York: McGraw-Hill, 2012:229-42.

3. Hokkanen L, Launes J, Michelsson K. Adult neurobehavioral outcome of hyperbilirubinemia in full term neonates-a 30 year prospective follow-up study. PeerJ 2014;2:e294.

4. Jangaard KA, Fell DB, Dodds L, Allen AC. Outcomes in a population of healthy term and near-term infants with serum bilirubin levels of $>$ or $=325$ micromol/L ( $>$ or $=19 \mathrm{mg} / \mathrm{dL})$ who were born in Nova Scotia, Canada, between 1994 and 2000. Pediatrics 2008;122:119-24.

5. Koziol LF, Budding DE, Chidekel D. Hyperbilirubinemia: subcortical mechanisms of cognitive and behavioral dysfunction. Pediatr Neurol 2013;48:3-13.

6. Tsuchie K, Miyaoka T, Furuya M, et al. The effects of antipsychotics on behavioral abnormalities of the Gunn rat (unconjugated hyperbilirubinemia rat), a rat model of schizophrenia. Asian J Psychiatr 2013;6:119-23.

7. Gunn $\mathrm{CH}$. Hereditary acholuric jaundice in a new mutant strain of rats. J Hered 1938;29:137-39.

8. Johnson L, Sarmiento F, Blanc WA, Day R. Kernicterus in rats with an inherited deficiency of glucuronyl transferase. AMA J Dis Child 1959;97(pt 1):591-608.

9. Schutta HS, Johnson L. Clinical signs and morphologic abnormalities in Gunn rats treated with sulfadimethoxine. J Pediatr 1969;75:1070-9.

10. Daood MJ, Hoyson M, Watchko JF. Lipid peroxidation is not the primary mechanism of bilirubin-induced neurologic dysfunction in jaundiced Gunn rat pups. Pediatr Res 2012;72:455-9.

11. Johnson L, Garcia ML, Figueroa E, Sarmiento F. Kernicterus in rats lacking glucuronyl transferase. II. Factors which alter bilirubin concentration and frequency of kernicterus. Am J Dis Child 1961;101:322-49.

12. Diamond I, Schmid R. Experimental bilirubin encephalopathy. The mode of entry of bilirubin-14C into the central nervous system. J Clin Invest 1966;45:678-89.
13. Rose AL, Wisniewski H. Acute bilirubin encephalopathy induced with sulfadimethoxine in Gunn rats. J Neuropathol Exp Neurol 1979;38: 152-64.

14. Shapiro SM, Hecox KE. Development of brainstem auditory evoked potentials in heterozygous and homozygous jaundiced Gunn rats. Brain Res 1988;469:147-57.

15. Butcher RE, Stutz RM, Berry HK. Behavioral abnormalities in rats with neonatal jaundice. Am J Ment Defic 1971;75:755-9.

16. Swenson RM, Jew JY. Learning deficits and brain monoamines in rats with congenital hyperbilirubinemia. Exp Neurol 1982;76:447-56.

17. Schutta HS, Johnson L. Bilirubin encephalopathy in the Gunn rat: a fine structure study of the cerebellar cortex. J Neuropathol Exp Neurol 1967;26:377-96.

18. Jew JY, Sandquist D. CNS changes in hyperbilirubinemia. Functional implications. Arch Neurol 1979;36:149-54.

19. Hebert MA, Gerhardt GA. Normal and drug-induced locomotor behavior in aging: comparison to evoked DA release and tissue content in fischer 344 rats. Brain Res 1998;797:42-54.

20. Stanford JA, Gerhardt GA. Age-related changes in striatal function of freely-moving F344 rats. Neurobiol Aging 2001;22:659-69.

21. Stanford JA, Gerhardt GA. Aged F344 rats exhibit altered electrophysiological activity in locomotor-unrelated but not locomotor-related striatal neurons. Neurobiol Aging 2004;25:509-15.

22. Hannigan JH Jr, Isaacson RL. The effects of Org 2766 on the performance of sham, neocortical, and hippocampal-lesioned rats in a food search task. Pharmacol Biochem Behav 1985;23:1019-27.

23. Lipska BK, Jaskiw GE, Weinberger DR. Postpubertal emergence of hyperresponsiveness to stress and to amphetamine after neonatal excitotoxic hippocampal damage: a potential animal model of schizophrenia. Neuropsychopharmacology 1993;9:67-75.

24. Wan RQ, Giovanni A, Kafka SH, Corbett R. Neonatal hippocampal lesions induced hyperresponsiveness to amphetamine: behavioral and in vivo microdialysis studies. Behav Brain Res 1996;78:211-23.

25. Naert A, Gantois I, Laeremans A, et al. Behavioural alterations relevant to developmental brain disorders in mice with neonatally induced ventral hippocampal lesions. Brain Res Bull 2013;94:71-81.

26. Blin O, Ferrandez AM, Serratrice G. Quantitative analysis of gait in Parkinson patients: increased variability of stride length. J Neurol Sci 1990;98: 91-7.

27. Koller WC, Trimble J. The gait abnormality of Huntington's disease. Neurology 1985;35:1450-4.

28. Goldfarb BJ, Simon SR. Gait patterns in patients with amyotrophic lateral sclerosis. Arch Phys Med Rehabil 1984;65:61-5.

29. Chaniary KD, Baron MS, Rice AC, Wetzel PA, Ramakrishnan V, Shapiro SM. Quantification of gait in dystonic Gunn rats. J Neurosci Methods 2009;180:273-7.

30. Graham DL, Hasenstab LL, Kinney L, Kuntz JA. The effects of litter size on the expressiviity of the jaundice genotype in Gunn rats. Dev Psychobiol 1980;13:55-60.

31. Stobie PE, Hansen CT, Hailey JR, Levine RL. A difference in mortality between two strains of jaundiced rats. Pediatrics 1991;87:88-93.

32. Keino H, Kashiwamata S. Critical period of bilirubin-induced cerebellar hypoplasia in a new Sprague-Dawley strain of jaundiced Gunn rats. Neurosci Res 1989;6:209-15.

33. Keino H, Aoki E, Kashiwamata S. Postnatal changes of the number and lobular distribution of acid phosphatase positive and lipid granule-containing cells in the cerebellum of hyperbilirubinemic Gunn rats. Neurosci Res 1986;3:183-95.

34. Molinari M, Petrosini L. Hemicerebellectomy and motor behaviour in rats. III. Kinematics of recovered spontaneous locomotion after lesions at different developmental stages. Behav Brain Res 1993;54:43-55.

35. Molinari M, Petrosini L, Gremoli T. Hemicerebellectomy and motor behaviour in rats. II. Effects of cerebellar lesion performed at different developmental stages. Exp Brain Res 1990;82:483-92.

36. Gould TJ, Bickford PC. The effects of aging on cerebellar beta-adrenergic receptor activation and motor learning in female F344 rats. Neurosci Lett 1996;216:53-6. 
37. Altabella L, Zoratto F, Adriani W, Canese R. MR imaging-detectable metabolic alterations in attention deficit/hyperactivity disorder: from preclinical to clinical studies. AJNR Am J Neuroradiol 2014;35:Suppl 6:S55-63.

38. Castellanos FX, Giedd JN, Marsh WL, et al. Quantitative brain magnetic resonance imaging in attention-deficit hyperactivity disorder. Arch Gen Psychiatry 1996;53:607-16.

39. Kessler RC, Berglund P, Demler O, Jin R, Merikangas KR, Walters EE. Lifetime prevalence and age-of-onset distributions of DSM-IV disorders in the National Comorbidity Survey Replication. Arch Gen Psychiatry 2005;62:593-602.
40. Wennberg RP, Ahlfors CE, Bhutani VK, Johnson LH, Shapiro SM. Toward understanding kernicterus: a challenge to improve the management of jaundiced newborns. Pediatrics 2006;117:474-85.

41. Schwartz HP, Haberman BE, Ruddy RM. Hyperbilirubinemia: current guidelines and emerging therapies. Pediatr Emerg Care 2011;27: 884-9.

42. Fowler SC, Birkestrand BR, Chen R, et al. A force-plate actometer for quantitating rodent behaviors: illustrative data on locomotion, rotation, spatial patterning, stereotypies, and tremor. J Neurosci Methods 2001;107: $107-24$. 